\title{
Optimizing Inductive Interconnect for Low Power
}

\author{
Magdy A. El-Moursy and Eby G. Friedman \\ Department of Electrical and Computer Engineering University of Rochester Rochester, New \\ York 14627-0231
}

\begin{abstract}
The width of an interconnect line affects the total power consumed by a circuit. A trade off exists between the dynamic power and the short-circuit power dissipated in inductive interconnect. The optimum line width that minimizes the total transient power dissipation is determined in this paper. A closed form solution for the optimum width with an error less than $5 \%$ is presented. For a specific set of line parameters and resistivities, a reduction in power approaching $78 \%$ is achieved as compared to the minimum wire width. Considering the driver size in the design process, the optimum wire and driver size that minimizes the total transient power is also determined.
\end{abstract}

Keywords: transient power dissipation,inductive interconnect, underdamped systems, shortcircuit power, characteristic impedance, dynamic power

\section{INTRODUCTION}

As the feature size of CMOS circuits and wiring has decreased, interconnect design has become an important issue in high speed, high complexity integrated circuits (IC). With the increase in signal frequencies and the corresponding decrease in signal transition times, the interconnect impedance can behave inductively [1], increasing the on-chip noise. Furthermore, considering inductance within the design process increases the computational complexity of IC synthesis and analysis tools. However, inductive behaviour can also be useful. As shown in [3], a properly designed inductive line can reduce the total power dissipated by high-speed clock distribution networks. Clock networks can dissipate a large portion of the total power dissipated within a synchronous IC, ranging from $25 \%$ to $75 \%[4,5]$. The technique proposed here can be used to reduce the overall power being dissipated by long interconnect such as a highspeed clock distribution network or data buss. 
Many algorithms have been proposed to determine the optimum wire size that minimizes a cost function such as delay [6] or power. Some of these algorithms address reliability issues by reducing clock skew. The work described in [7] considers simultaneous driver and wire sizing using the Elmore delay model with simple capacitance, resistance, and power models. As the inductance becomes important, certain algorithms have been enhanced to consider an RLC model [8].

In this paper, the tradeoff between short-circuit and dynamic power in inductive interconnect is introduced. The optimum line width that minimizes the total power dissipation is determined. As the line driver has an important effect on the signal and power dissipation characteristics, a closed form solution for the simultaneous driver and wire-sizing problem that minimizes the total transient power dissipation is presented. An analytical solution for the transition time at the far end of a long inductive interconnect is also provided. These results are used to determine a closed form solution for the width of an inductive interconnects line that minimizes the power dissipated by that line.

The paper is organized as follows. In section 2, the transient power characteristics of inductive interconnect line are discussed. The signal behaviour at the end of an inductive line is described in section 3. In section 4, a power optimization criterion is formulated. The effect of line material and length on the optimum interconnect width is demonstrated in section 5. Some conclusions are discussed in section 6 .

\section{POWER CHARACTERISTICS OF INDUCTIVE INTERCONNECT}

The transient powers characteristics of inductive interconnect are presented in this section. The research described [3] uses wire sizing techniques to reduce the total transient power dissipated by a clock distribution network, but does not provide a closed form solution to determine the optimum interconnect width. Their model also ignores the change in the circuit behavior that occurs when the width of the line is increased. The matching response between the line and the driver plays an important role in the transient power dissipation as discussed in section 3. In [3], the driver size is also not considered as a design parameter.

Issues that affect wire sizing are discussed in this section. In subsection 2.1, the trade off between dynamic and short-circuit power dissipation in inductive interconnect is described. A lossy transmission line model used in the development of a closed form solution for the interconnect width that minimizes the total transient power is presented in subsection 2.2. Simultaneous wire and driver sizing criteria are described in subsection 2.3. 
A trade-off exists between dynamic and short-circuit power in sizing inductive interconnect. As shown in Fig1, an optimum interconnect width at

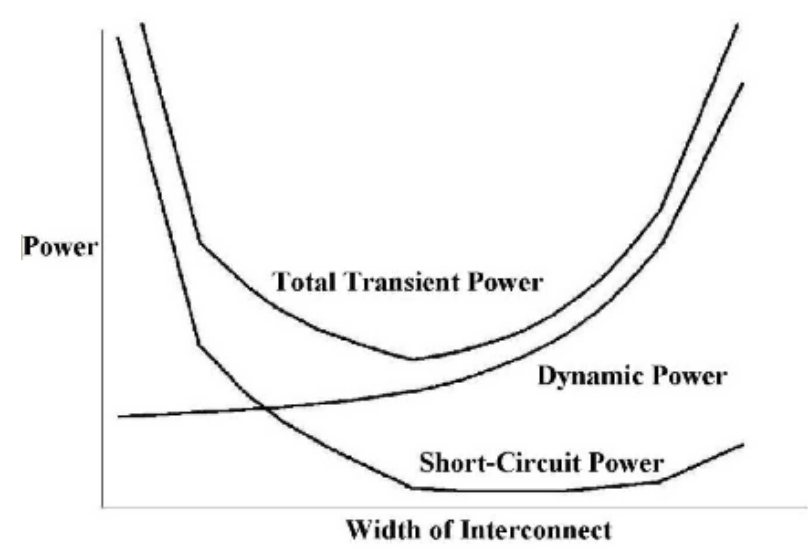

Figure.1: Dynamic, short-circuit, and total transient power as a function of the connect line width

which the total transient power is a minimum exists for overdriven lines. This trade off does not occur if the line is under driven, as described in section 3.

Transient power dissipation is composed of dynamic power and short-circuit power [2]. For the circuit shown in Fig. 2, both Inv1 and Inv2 dissipate transient power during switching. The change in the line width primarily affects the dynamic power of Inv1.P1d and the short-circuit power of Inv2 P2sc Closed form expressions for the dynamic and short-circuit power are given by (1) and (2), respectively [9]. The dynamic power of Inv2 depends on the load capacitance, and is not affected by the wire size. The change in the short-circuit power of Inv1 is negligible, assuming a fixed signal transition time at the input of Inv1

$$
\begin{aligned}
& \mathrm{P}_{\mathrm{id}}=\mathrm{fV}_{\mathrm{dd}}^{2} \mathrm{C}, \\
& \mathrm{P}_{2 \mathrm{sc}}=\mathrm{f}(1 / 12) \mathrm{k}_{2} \tau_{\mathrm{o}}\left(\mathrm{V}_{\mathrm{dd}}-2 \mathrm{~V}_{\mathrm{t} 2}\right)^{3}
\end{aligned}
$$

Where $\mathrm{f}$ is the operating frequency, $\mathrm{C} 1$ is the total capacitance driven by Inv1, $\mathrm{K}_{2}$ and $\mathrm{V}_{\mathrm{t}_{2}}$ are the trans conductance and threshold voltage of $\mathrm{Inv}_{2}$, respectively, and $\tau_{\mathrm{o}}$ is the transition time of the signal at the input of $\operatorname{Inv}_{2}$. 


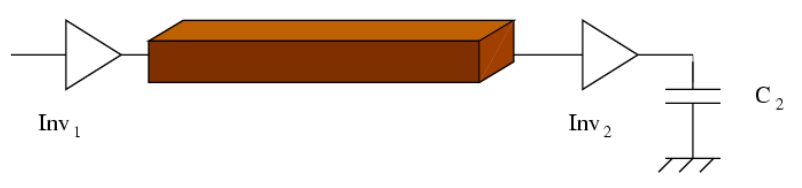

Figure.2: CMOS inverter driving another inverter through a long interconnect line

Only $\mathrm{C}_{1}$ and $\tau_{0}$ are affected by a change in the line width. Changing the line width has a significant effect on the transition time, as described in section 3 . To determine the optimum width, closed form expressions for both $C_{1}$ and $\tau_{0}$ are provided in subsections 2.2 and 3.1 , respectively.

\section{2}

\section{Transmission Line Model}

To obtain a closed form solution for the optimum width, expressions for the line impedances which model the interconnect are presented. Neglecting the line dielectric losses, a lossy transmission line is represented by the line resistance $\mathrm{R}$, inductance $\mathrm{L}$, and capacitance $\mathrm{C}$, all per unit length. R, L, and C are expressed in terms of the line dimensions in (30-(5) respectively.

$$
\mathrm{R}=\rho / \mathrm{W}_{\text {int } \mathrm{T}}
$$

where $\rho, \mathrm{T}$ and $\mathrm{W}_{\mathrm{int}}$ are the line resistivity, thickness, and width, respectively. Assuming a single line, $\mathrm{C}$ is given by [16]

$$
\frac{C}{\epsilon_{o x}}=1.13 \frac{W_{I N T}}{H}+1.44\left(\frac{W_{I N T}}{n}\right)^{0.11}-1.47\left(\frac{T}{H}\right)^{0.42}
$$

Note that $\mathrm{C}$ increases super linearly with the line width, which increases the dynamic power $P_{1 d .}$. is the self-inductance of the line and is given by [12]

$$
L=200\left(\ln \left(\frac{2 l}{W_{I N T}+T}\right)+0.5+0.22 \frac{W_{I N T}+T}{l}\right)
$$

The self-inductance decreases with increasing line width. Note that the line inductance may increase if the total area of the interconnect is fixed, since the mutual inductance can increase with smaller line-to-line spacing. For a single line, however, the interconnect inductance decreases with increasing width.

The inductive time constant of a line can be characterized by $\sqrt{ } 1 / R[10]$. 
This time constant increases with increasing line width, as the reduction in resistance is much greater than the reduction in the inductance. An increase in the inductive time constant and line capacitance affects the transition time as described in section 3.

\subsection{Simultaneous Wire and Driver Size}

The geometric width of the driver also plays an important role in the total transient power dissipation. Two complementary effects occur. As the driver size increases, the transition time of the output signal decreases and, consequently, the short-circuit power of the load gate decreases. At the same time, the driver gate input capacitance increases as the width of the driver becomes larger, increasing the power required to charge the gate capacitance. A general expression for the total transient power dissipation including the power dissipation due to the driver input gate capacitance is

$$
\mathrm{P}_{\text {tdrive }}=\mathrm{P}_{\text {id }}+\mathrm{P}_{2 \mathrm{sc}}+\mathrm{P}_{\text {drive }}
$$

Where $\mathrm{P}_{\text {drive }}=\mathrm{f}_{\mathrm{dd}}^{2} \mathrm{C}_{\text {drive }}$ and $\mathrm{C}_{\text {drive }}$ is the driver gate input capacitance. Equation (6) is therefore a function of both the wire width and the driver size. To achieve the global minimum for the transient power dissipation, both of these design variables need to be simultaneously determined.

\section{TRANSITION TIME FOR A SIGNAL AT THE FAR END OF AN INTERCONNECT LINE}

Wire sizing techniques do not consider line-matching characteristics as the line width changes. For inductive interconnect, the matching response plays an important role in the signal characteristics. It is shown in this section that, for an under driven line, the transition time increases as the line becomes wider. A closed form expression of the signal transition time at the far end of a line is presented in subsection 3.1.The effect of wire sizing on the line matching characteristics and the transition time is described in subsection 3.2.

\section{$3.1 \quad$ CLOSED FORM EXPRESSION FOR THE TRANSITION TIME}

To determine a closed form expression for the high-to-low signal transition time at the far end of an inductive interconnect line, a lumped RLC equivalent circuit is used to 
model the interconnect impedance [2]. Assuming the PMOS transistor of the driving inverter is off, an analytical expression for the signal at the far end of the line is

$$
\mathrm{V}(\mathrm{t})=\mathrm{V}_{\mathrm{c}}\left(\tau_{\mathrm{p}} \mathrm{OFF}\right) \mathrm{e}^{- \text {an(t- } \tau \text { off })}
$$

Where $\tau_{\mathrm{p}} \mathrm{OFF}$ is the time at which the PMOS transistor of the driver turns off, and $\alpha_{\mathrm{n}}$ is a constant that depends upon R, C, L, and the driver transistor characteristics such as the transconductance, mobility, and threshold voltage. $\mathrm{V}_{\mathrm{c}}\left(\tau_{\mathrm{p}} \mathrm{OFF}\right)$ is the voltage at the load capacitance at $\tau_{\mathrm{p}} \mathrm{OFF}$. The transition time is expressed by $\tau_{0}=\mathrm{t}$ $10 \%-t_{90 \%}$ where $t_{10 \%}$ and $t_{90 \%}$ are the times at which the signal reached $10 \%$ and $90 \%$ of the final value, respectively.The transition time based on this analytical expression is compared to SPICE in Fig. 3

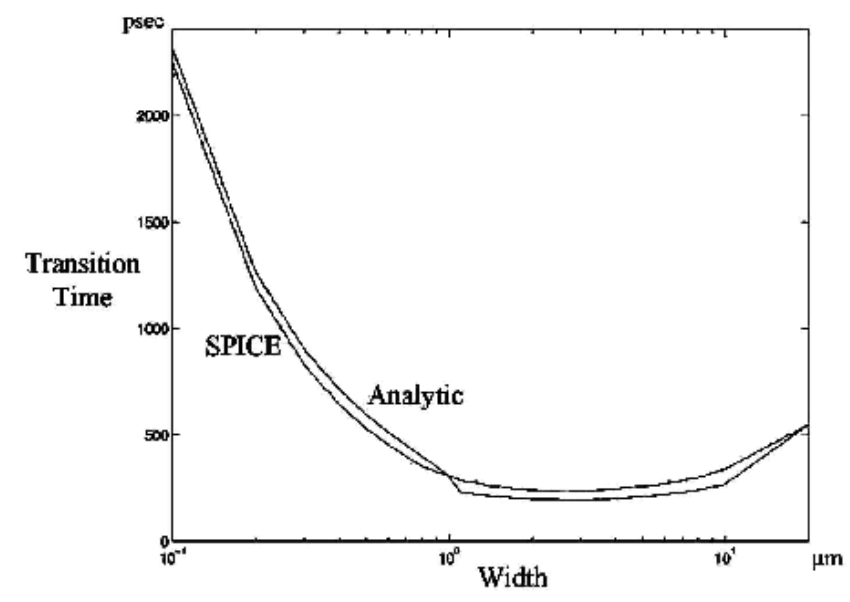

Figur.3: Analytical solution of the trasistion time as compared with SPICE for different line widths

The values of R, C and L are determined from (3) -(5) based on $\mathrm{H}=2 \mu \mathrm{m}$, T $=2 \mu \mathrm{m}, \rho=2.5 \mu \Omega \mathrm{cm}$ and $\mathrm{l}=5 \mathrm{~mm}$. A $0.24 \mu \mathrm{m}$ CMOS inverter with $\mathrm{W}_{\mathrm{n}}=15 \mu \mathrm{m}$ and $\mathrm{W}_{\mathrm{p}}=30 \mu \mathrm{m}$ is assumed.The maximum error in the analytical expression as compared to SPICE is less than $20 \%$ and is typically around $14 \%$.

As shown in Fig3, as the line width increases, the signal transition time decreases until a minimum transition time is reached. The signal transition time increases again after exceeding a certain line width. The behavior of the transition time in terms of the line impedances is described in the following section. 


\subsection{EFFECTS OF CHANGING THE INTERCONNECT WIDTH ON THE LINE CHARACTERISTICS}

To describe the signal behavior in terms of the interconnect width, an equivalent circuit of an inverter driving an inductive interconnect line is used. The characteristic impedance of a lossy line is given by the well-known formula $Z_{\text {lossy }}=\sqrt{ } R+j w L / j w C$. The input signal driving the line is assumed to be a ramp signal. Different approximations have been made to estimate $Z_{\text {lossy }}$ in terms of the per unit length parameters $[14,15]$. A general form for $Z_{\text {lossy }}$ is $Z_{o}+g R$ where $g$ is a constant which depends on the line parameters.

For the high-to-low input transition, the NMOS transistor is assumed to be off. The inverter can be replaced with an ideal voltage source with a variable output resistance $\mathrm{R}_{\text {tr }}$ as shown in Fig 4.

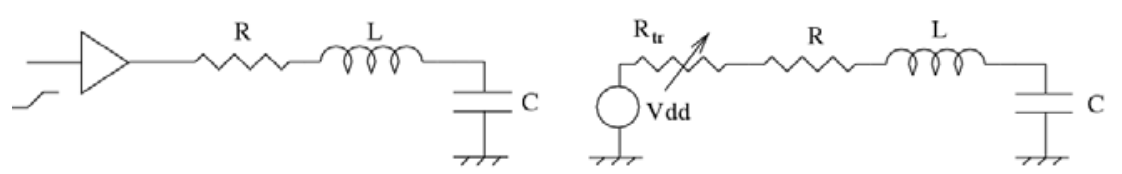

Figure.4: Equivalent circuit of an inverter driving an $R L C$ interconnect line

At small interconnect widths; the characteristic line impedance is large as compared to the equivalent output resistance of the transistor. Thus, the line is overdriven and the waveform at the far end of the line contains overshoots and undershoots (the under damped condition ). $Z_{\text {lossy }}$ decreases with increasing line width, and the line remains under damped until $Z_{\text {lossy }}$ equals $\mathrm{R}_{\text {tr.. }}$ A further increase in the line width under drives the line, as $Z_{\text {lossy }}$ becomes less than $R_{\text {tr }} \quad$ [13]. As the line width is increased, the line driving condition changes from overdriven to matched to under driven.

For an overdriven line, increasing the line width makes the line more inductive as the resistance decreases linearly while the inductance has a logarithmic dependence on the width (for a single line). As described in [3], the line impedance approaches becoming lossless, and the attenuation constant approaches zero at large line widths. This effect reduces the signal transition time. A further increase in the line width decreases $Z_{\text {lossy }}$ and matches the line impedance with the driver impedance. At this width, the transition time reaches a minimum, as shown in Fig 3.Increasing the width further under drives the line. At this width, the capacitance begins to dominate the line impedance. The line becomes highly capacitive which further increases the transition time, increasing the short-circuit power dissipation in the load inverter. For an overdriven line, the short-circuit power dissipation changes with the line width as shown in Fig. 1. However, for an under driven line, an increase in the line width increases the short-circuit power. If the line is under driven, the line should be made as thin as possible to minimize the total transient power. 


\section{INTERCONNECT WIDTH OPTIMIZATION FOR MINIMUM POWER}

Using the closed form expressions for $\mathrm{C}$ and $\tau_{o}$, the transient power components can be expressed in terms of the line width. A criterion for determining the optimum interconnect width is applied in this section to a simple example circuit and compared with SPICE.

The dynamic power of a driver increases with the interconnect width as the line capacitance increases. The short-circuit power within the load gate decreases as the line becomes wider (more inductive). When the width reaches the matched condition, the short-circuit power is minimum. An increase in the interconnect width beyond the matching condition increases the short-circuit power, since the transition time increases (see Fig. 3). For an inverter driving $\mathrm{N}$ gates, as shown in Fig. 5a, the total transient power is given by

$$
\mathrm{P}_{\text {tDrive }}=\mathrm{P}_{\text {tDrive }}+\mathrm{N} \mathrm{P}_{2 \mathrm{sc}}+\mathrm{P}_{\text {drive }}
$$

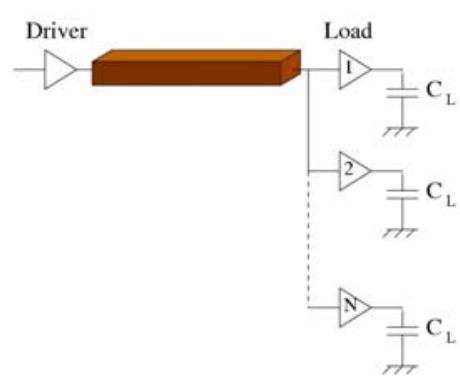

(a)

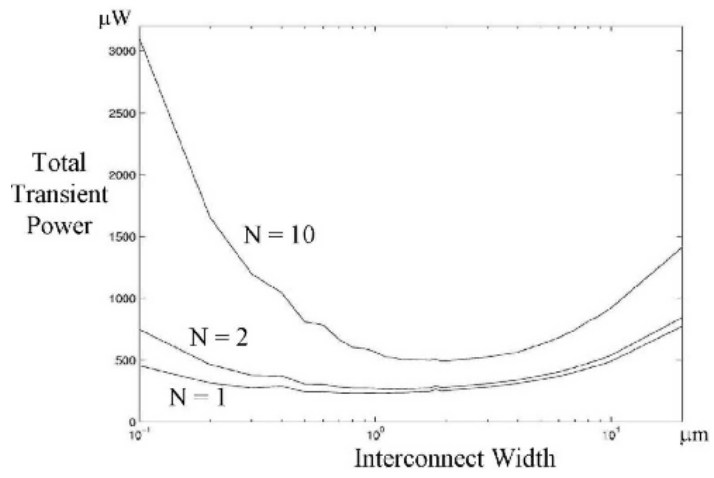

(b)

Figure.5: Inverter driving $\mathrm{N}$ logic gates, a 0 circuit structure, b0 total transient power dissipation as determined by SPICE

For a specific driver size, the total transient power dissipation is a function of the line width. Given a set of line parameters, the optimum line width can be obtained by determining the interconnect width which minimizes $\mathrm{P}_{\text {tDrive }}$. For circuit specifications as described in subsection 3.1, the total simulated power dissipation for different loads, $\mathrm{N}=1,2$,and 10, is shown in Fig. 5b. A comparison between the analytic solution and simulation is listed in Table 1. The error between the analytic solution and SPICE for the chosen range of values is less than $5 \%$. 


\begin{tabular}{|l|l|l|l|}
\hline $\begin{array}{l}\text { Number of Loads } \\
\mathrm{N}\end{array}$ & \multicolumn{2}{|l|}{$\mathrm{W}_{\text {INT Optimum }}(\mu \mathrm{m})$} & Error (\%) \\
\hline & Analytical & SPICE & \\
\hline 1 & 1.08 & 1.10 & -1.9 \\
2 & 1.13 & 1.15 & -1.8 \\
10 & 1.99 & 1.90 & +4.8 \\
\hline
\end{tabular}

For $\mathrm{N}=10$, the total transient power dissipation of a symmetric driver is shown in Fig6. Considering the driver size as a design variable, a different local minimum for the transient power dissipation exists for each driver size. Furthermore, for each line width, a minimum transient power dissipation also exists for each driver size. A global minimum for the transient power is obtained by determining the optimum value of each design variable. Considering the driver size as a design parameter, (8) is a function of two variables, permitting the global minimum for the power dissipation to be obtained. For the example circuit shown in Fig. 5a, the minimum power is achieved at $\mathrm{W}_{\mathrm{INT}}=$ $2.8 \mu$ mand $\mathrm{W}=57 \mu \mathrm{m}$.

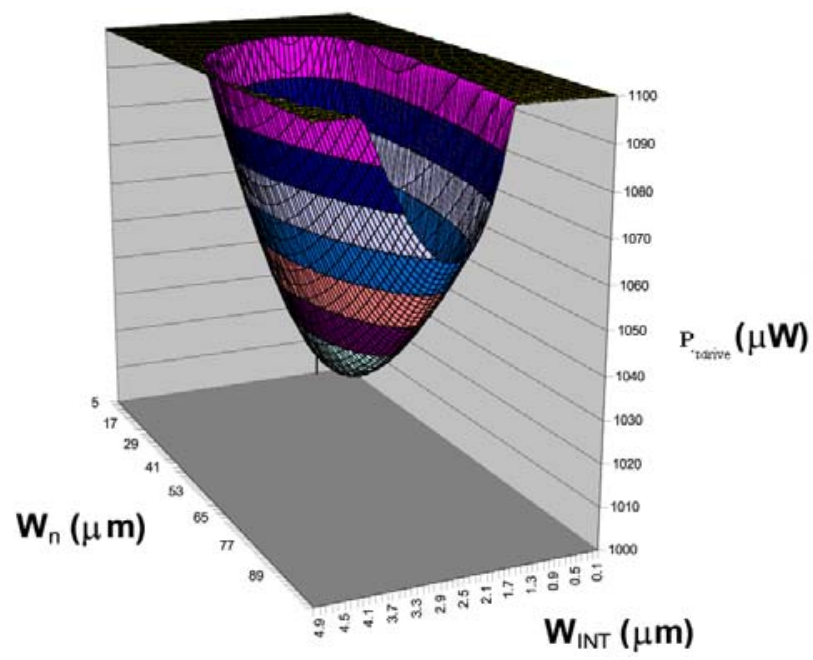

Figure.6: Total power dissipation with different wire and driver sizes where N=10.

\section{EFFECTS OF INTERCONNECT RESISTIVITY AND LENGTH ON POWER}

To evaluate the effectiveness of the optimum solution, different interconnect line parameters are considered. To demonstrate the importance of the optimum solution 
on inductive lines, the optimum width is obtained for two line lengths, $1=1 \mathrm{~mm}$ and 5 $\mathrm{mm}$. The transient power dissipation is determined for three line widths, thin, optimum, and wide. As listed in Table 2, the optimum width of a copper line reduces the total transient power by $68.5 \%$ for $1=5 \mathrm{~mm}$ as compared to $28.6 \%$ for $1=1 \mathrm{~mm}$. For aluminium, a reduction of $77.9 \%$ is achieved as compared to $37.8 \%$. As the line become longer (i.e. more inductive), the power decreases further. The more inductive the interconnect, the more sensitive the power dissipation is to a change in the line width. Wire width optimisations is, therefore, more effective for longer inductive lines.

Also listed in Table 2 is that the power reduction in aluminium is greater than that in copper. The reduction in power in these inductive lines increases further as the lines become more resistive (see, for example, aluminium lines versus copper lines in Table 2.)Comparing the optimum width with the minimum width, the reduction in power is greater in more highly resistive lines.

\begin{tabular}{|c|c|c|c|c|c|}
\hline $\begin{array}{c}\text { Resistivity } \rho \\
\mu \Omega \mathrm{cm}\end{array}$ & \multicolumn{5}{|c|}{ Total Transient Power Dissipation $(\mu \mathrm{W})$} \\
& \multicolumn{5}{|c|}{ Resistive line(I=1 mm) } \\
\hline & Optimum & Thin & Reduction & Wide & Reduction \\
\hline & 583 & 817 & $28.6 \%$ & 880 & $33.8 \%$ \\
\hline 1.7 (copper) & 606 & 976 & $37.8 \%$ & 813 & $25.4 \%$ \\
\hline 2.5 (Aluminium) & \multicolumn{5}{|c|}{ Inductive Line $(\mathrm{I}=5 \mathrm{~mm})$} \\
\hline & 1121 & 3563 & $68.5 \%$ & 1931 & $41.9 \%$ \\
\hline 1.7 ( copper) & 1236 & 5592 & $77.9 \%$ & 1973 & $37.4 \%$ \\
\hline 2.5 (Aluminium) & \multicolumn{5}{|c|}{}
\end{tabular}

\section{CONCLUSION}

It is shown in this paper that a trade off exists between the dynamic and shortcircuit power in inductive inter connect. The short-circuit power of an overdriven interconnect line decreases with the line width, while the dynamic power increases. When the line exceeds the matched condition, the short-circuit power also increases with increasing line width. For a long inductive interconnect line, an optimum interconnect width exists that minimizes the total transient power dissipation. A closed form solution is presented for determining this optimum width. This solution has high accuracy, producing an error of less than 5\%. The optimum line width is shown to be more effective in reducing the total transient power as the line becomes longer. With aluminum interconnect, a reduction in power of about $80 \%$ and $37 \%$ is obtained as compared to thin and wide wires, respectively. For copper interconnect, a reduction in power of $68 \%$ and $42 \%$ is obtained for the same conditions. Greater power reduction is achieved for optimally sized lines with higher resistively as compared to minimum 
width lines. The optimum interconnect width depends upon both the driver size and the number of gates being driven. With this solution, the optimum driver and wire size can be simultaneously determined.

\section{REFERENCES}

[1] Y. I. Ismail, E. G. Friedman, and J. L. Neves, "Figure of Merit to Characterize the Importance of On-Chip Inductance," IEEE Transactions on Very Large Scale Integration (VLSI) Systems\}, Vol. 7, No. 4, pp. 442-449, December 1999.

[2]K. T. Tang and E. G. Friedman, "Delay and Power Expressions Characterizing a CMOS Inverter Driving an RLC Load," Proceedings of the IEEE International Symposium on Circuits and Systems\}, Vol. III, pp. 283-286, May 2000

[3]Y. I. Ismail, E. G. Friedman, and J. L. Nerves, "Exploiting On-Chip Inductance in High Speed Clock Distribution Networks," IEEE Transactions on Very Large Scale Integration (VLSI) Systems \}, Vol. 9, No. 6, pp. 963 - 973, December 2001.

[4]P. E. Gronowski et al., "High-Performance Microprocessor Design," IEEE Journal of SolidState Circuits\}, Vol. 33, No. 5, pp. 676-686, May 1998.

[5]C. J. Anderson, et al., "Physical Design of a Fourth-Generation POWER GHz Microprocessor," Proceedings of the IEEE International Solid-State Circuits Conference, pp. 232-233, February 2001.

[6]J. J. Cong, K. Leung, and D. Zhou, "Performance-Driven Interconnect Design Based on Distributed RC Delay Model, "IEEE Transactions on Computer-Aided Design of Integrated Circuits and Systems, Vol. 14, No. 3, pp. 321-336, March 1995.

[7]J. J. Cong and C.-K. Koh, "Simultaneous Driver and Wire Sizing for Performance and Power Optimization," IEEE Transactions on Very Large Scale Integration (VLSI) Systems\}, Vol. 2, No. 4, pp. 408-425, December 1994.

[8]Q. Zhu and W. M. Dai, "High-Speed Clock Network Sizing Optimization Based on Distributed RC and Lossy RLC Interconnect Models, "IEEE Transactions on Computer-Aided Design of Integrated Circuits and Systems, Vol. 15, No. 9, pp. 1106-1118, September 1996.

[9]H. J. M. Veendrick, "Short-Circuit Power Dissipation of Static CMOS Circuitry and its Impact on the Design of Buffer Circuits," IEEE Journal of Solid-State Circuits\}, Vol. SC-19, No. 4, pp. 468-473, August 1984.

[10]Y. I. Ismail and E. G. Friedman, "Effects of Inductance on the Propagation Delay and Repeater Insertion in VLSI Circuits, "IEEE Transactions on Very Large Scale Integration (VLSI) Systems, Vol. 8, No. 2, pp. 195 - 206, April 2000.

[11] X. Qi, G. Wang, Z. Yu, R. W. Dutton, T. Young, and N. Chang, "On-Chip Inductance Modelling and \$RLCS Extraction of VLSI Interconnects for Circuit Simulation," Proceedings of the IEEE Custom Integrated Circuits Conference\}, pp. 487-490, May 2000.

[12]E. B. Rosa, "The Self and Mutual Inductances of Linear Conductors, "Bulletin of the National Bureau of Standards, Vol. 4, No. 2, pp. 301-344. Government Printing Office, Washington, January 1908.

[13]J. Wang and W. Dai, "Optimal Design of Self-Damped Lossy Transmission Lines for Multichip Modules," Proceedings of the IEEE International Conference on Computer Design, pp. 594-598, October 1994.

[14]Cappuccino and G. Cocorullo, "A Time-Domain Model Power Dissipation of CMOS Buffer Driving Lossy Lines,"Electronics Letters, Vol. 35, No. 12, pp. 959-960, June 1999.

[15]P.Heydari and M. Pedram, "Calculation of Ramp Response of Lossy Transmission Lines Using Two-Port Network Functions," Proceedings of the ACM International Symposium on Physical Design, pp. 152-157, April 1998.

[16]N. Delorme, M. Belleville, and J. Chilo, "Inductance and Capacitance Analytic Formulas for VLSI Interconnects," Electronics Letters, Vol. 32, No. 11, pp. 996-997, May 1996. 